\title{
Medicine Scheduler Using Node MCU
}

\author{
Dr.(Mrs) Punam Chati ${ }^{1}$, Ms. Aishwarya Kapse ${ }^{2}$, Mr. Aniruddha Dhabarde ${ }^{3}$,Ms.Aditi Vairagade ${ }^{4}$, \\ Ms.Vaishali Akre 5 \\ ${ }^{1}$ Assistant Professor, ${ }^{2,3,4,5,6}$ Students Research Scholar, \\ Department of Electronics and Telecommunications, Priyadarshini College of Engineering, Nagpur, India
}

DOI: 10.46335/IJIES.2020.5.10.9

\begin{abstract}
The idea of a digital world where different types of sensors and local processing connected to share information is used in many industries nowadays. There are various products which are developed based on these ideas. Healthcare industry is one where a lot of improvements are taking place. Medicines play an important role for prevention and cure for most of the diseases. Many Harmful and risky diseases can be cured through proper medication. The proposed system consists of an IoT enabled medication reminder system and it gives timely alerts for the patients about their medication time. It alerts the patient to take medicines at the proper time by providing audio-visual alert. The system helps to monitor whether a patient has taken the medicine and it's healthcare data.
\end{abstract}

Keywords- Automatic Alarm, Reminder System, Notification System, Medication Adherence, Medicine.

\section{I- INTRODUCTION}

$\mathbf{T}$ he idea of a digital world where different types of sensors and local processing connected to share information is used in many industries nowadays. There are various products which are developed based on these ideas. Healthcare industry is one where a lot of improvements are taking place.

Medicines play an important role for prevention and cure for most of the diseases. Many Harmful and risky diseases can be cured through proper medication. The proposed system consists of a medication reminder system and it gives timely alerts for the patients about their medication time. It alerts the patient to take medicines at the proper time by providing a buzzer alert. The system helps to monitor whether a patient has taken the medicine and it's healthcare data.
In today's life, everyone has to take medicines due to several health issues. In olden days this problem was less compared to now. Well, everyone does forget to take their medicines on time at some point in their life and it is very important to take all the medicines properly and on time. Similarly, taking medicine at wrong intervals may lead to some severe side effects. Some diseases are temporary so the person need not take medicine every day but there are many everlasting life threatening diseases. These life- threatening diseases are very dangerous to the health, so that kind of people have to take their medicine daily without fail. Blind people and adults cannot always remember to take the medicine regularly which creates several problems and health issues. We need to have the counsel of Doctor who tells us to take desired pills in a desired way so that patients face problems like forgetting pills at the right time. Since the target audience of the device is Geriatric, it is user-friendly, handy, safe to use and light in weight. It is a portable device and it is a handheld system. Blind people, uneducated people can also use this system. There is no age limit to use this device

\section{II- EXISTING SYSTEM}

The board we are using is called "Node MCU" and has an ESP8266 module on it, which we will be programming. It comes with the latest version of Micro Python already setup on it, together with all the drivers we are going to use. The D0, D1, D2, ... numbers printed on the board are different from what Micro python uses - because originally those boards were made for a different software. Espressif's ESP8266EX delivers a highly integrated Wi-Fi SoC solution to meet users continuous demands for efficient 
power usage, compact design and reliable performance in the Internet of Things industry. With the complete and selfcontained Wi-Fi networking capabilities.

\section{III- PROPOSE SYSTEM}

The users will get the notifications through SMS also. It will provide the information about the medicine timings. The scheduled appointment with the doctor with the contact details including visiting time, venue and availability at different hospitals in case the appointment is missed at the scheduled place. The new appointment will be set accordingly.t. The system focuses on improving the rate of attendance at healthcare appointments. The personal phone notifications and reminders are a strong supporting tool in improving medication adherence strategies. It supports an easy implementation as it is less expensive, reliable, scalable, accessible to anyone with smartphones, and does not require separate devices, packaging or extra hardware. In case if the user's phone is switched off and he has set the alarm and the notification is set on, still he will be able to get the notifications through email or message(on his device as well as on other registered numbers), so it works even when you are running out of battery. Also a facility of reminding the doctor's next appointment in the system has been focussed.We have also implemented a navigation system which will allow users to locate the nearest registered hospitals according to their current location.

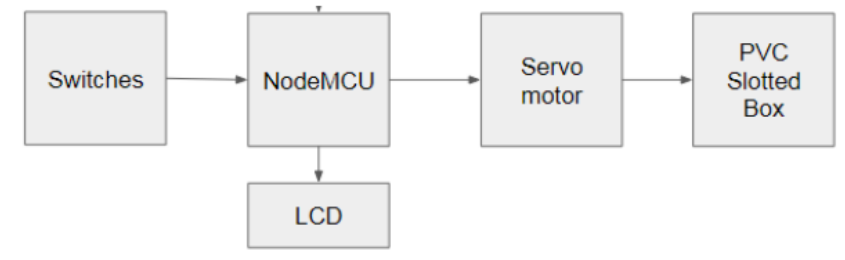

Figure 1: System Block Diagram

The location based searching of the doctors as well as disease wise searching has been focused which makes the application more suitable, more user friendly with great features and satisfactory results.

\section{IV-METHODOLOGY}

We can set the timings between the schedule of medicine taking by using buttons.LCD will display to set the timings. Servo motor will rotate the box of medicine as per the schedule When the slot open for the medicine and if the medicine is taken out from the box the signal is send to cloud server to update the database that the medicine is taken out w.r.t date and time and vice versa Node MCU is programmed using Arduino software and to communicate with the cloud server

\section{Requirement Analysis Hardware}

1. NodeMCU: The board we are using is called "NodeMCU" and has an ESP8266 module on it, which we will be programming

2. LCD Display: for displaying special \& even custom characters (unlike in seven segments), animations. The status of the system is displayed using LCD

3. Servo Motor : It consists of a suitable motor coupled to a sensor for controlling the angular or linear position

4. IR sensor:to sense some aspects of the surroundings and to measure the heat of an object as well as detects the motion

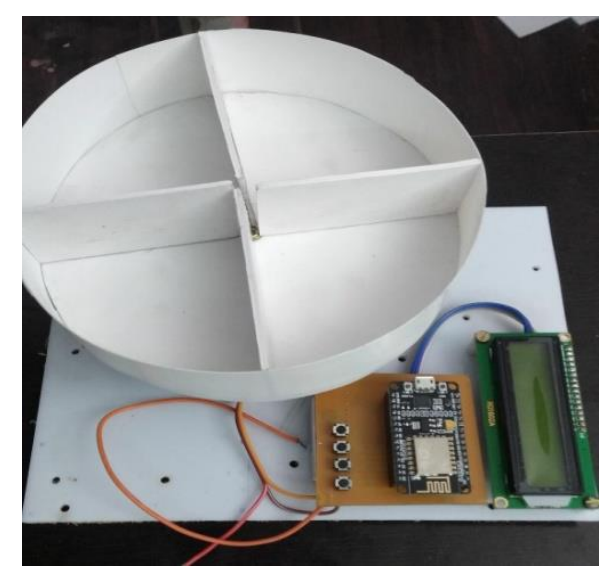

Fig 2-Medicine Scheduler using NodeMCU

Hardware components for augmented reality are: processor, display, sensors and input devices. Acrylic sheet is transparent shit and it is easy to show the reflection of any light source. All the components are in PCB and fit in a box.

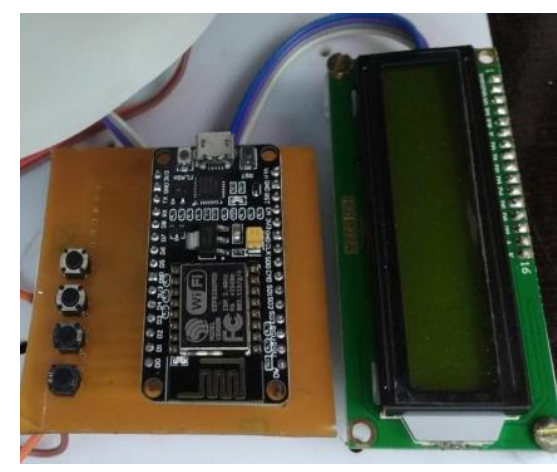

Fig 3- Medicine Scheduler using NodeMCU 
When you are satisfied with your schematic design and simulation results, you can then quickly proceed to produce your finished and populated PCB without leaving EasyEDA. EasyEDA's PCB Design canvas helps you to quickly and easily lay out even complex multilayer designs from schematics you have already created in the Schematic canvas or directly as a layout with no schematic and position to the database then database will fetch the requirements sent by the user and then it shows the destination to the user.

\section{Software:}

- Arduino IDE compiler: The Arduino IDE supports the languages $\mathrm{C}$ and $\mathrm{C}++$ using special rules of code structuring

- EasyEDA: It is a web-based EDA tool suite that enables hardware engineers to design, simulate, share -publicly and privately - and discuss schematics, simulations and printed circuit boards.

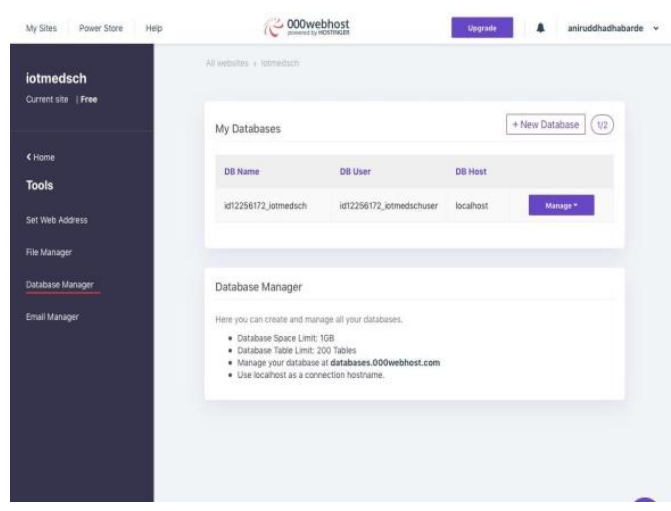

Fig 3- Arduino IDE compiler

- $\quad \mathrm{C}$ and $\mathrm{C}++$ :These are programming languages and used for application development.

- Cloud Server:For our project we have used 000webhost.com as a free web cloud server.

\section{V- FUTURE WORK AND CONCLUSION}

In today's market there are many medication intake reminder systems available. These systems have been made on various different platforms. Many of these systems require special hardware devices to perform the services of medication intake reminder. Thus in our project we have attempted creating a system which is easily accessible and is also economical for providing timely reminders.

\section{REFERENCES}

[1] "Smartphone medication adherence apps: Potential benefits to patients and providers", available at: https://www.ncbi.nlm.nih.gov/pmc/articles/PMC3919626/

[2] "Alarm Pill Box Pill Mate Event Reminder", https://www.amazon.com/Alarm-Pill-Mate-Eventreminder/dp/B 000R\%203EETI

[3] Smart Medicine Reminder with Smart Watch Using Arduino Nano, International Journal of Research and Scientific Innovation (IJRSI) | Volume VI, Issue IV, April 2019

[4] Auto Pill Dispenser, IJSRD - International Journal for Scientific Research \& Development| Vol. 6, Issue 01, 2018 ISSN (online): 2321-0613 